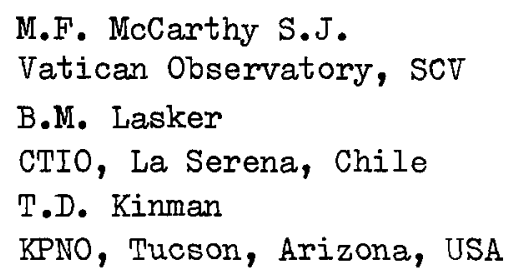

V 4049 Sgr was detected by the authors as a possible symbiotic star on Curtis Schmidt objective prism plates. This star is Nova Sgr 1978 and was first discovered by Stenholm and Lindstrom. When observed in 1979 with SIT specgrograph on CTIO $4 \mathrm{~m}$ telescope the outstanding features were the presence of very strong lines of [FeVII] chiefly at $6085 \mathrm{~A}$ where its flux relative to $\mathrm{H}_{\alpha}$ is 1.6. Details have been published in Pub. astr. Soc. Pacific, 23, 470 (1981).

\title{
DISCUSSION ON RR TELESCOPII
}

Slovak: Have Warner or Walker pursued high speed photometry of $\mathrm{RR} \mathrm{Tel}$, and if so, what has been found?

Whitelock: I do not think that Walker detected flickering in RR Tel. Penston et al. discussed flickering detected by Stromgren photometry.

Cassatella: I think that these observations need confirmation befo re we can say that flickering has definitely detected.

Friedjung: I would like to make three comments:

1) The high velocity wings seen in the UV remind me of the Wolf Rayet profiles seen by Webster in the visual. I would suspect this to be due to a wind from the hot component.

2) I object slightly to one talking of a normal D type symbiotic star. The kinematics studied by Thackeray is complex. This is a unique animal! 3) L. A. Willson in a talk given in Boulder concluded that the Mira com ponents of $D$ type symbiotics were quite normal. 
Michalitsianos: There appears to be a relationship between mass loss rate and pulsation period in single Miras from infrared work. Accordingly, if symbiotics contain Miras of longer periods, say $P>350$ days, the interaction between components in the system may be enhanced due to higher mass loss rates.

Kafatos: It is puzzling that in RX Pup in particular, where the $1551 / 1548$ ratio is reversed (about $2: 1$ ) with respect to the theoretical ratio, this would indicate the only evidence of broad P Cygni profiles in the IUE range $\left(\sim 600 \mathrm{~km} \mathrm{~s}^{-1}\right)$, i.e. the 1551 P Cygni absorption pulls down the 1548 emission. 